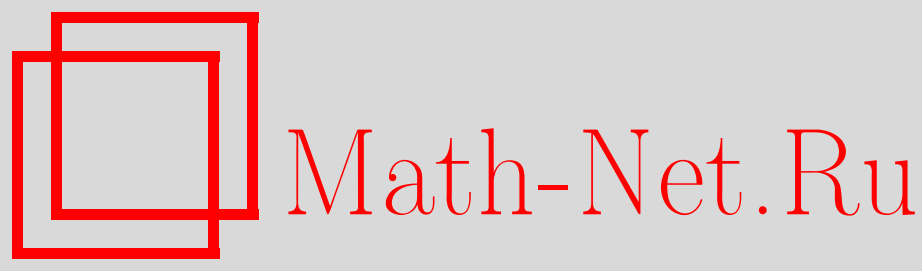

А. А. Кокин, В. А. Кокин, О косвенном взаимодействии между ядерными спинами в легкоосном антиферромагнетике, ТМФ, 2011, том 168, номер 3, 467-481

DOI: https://doi.org/10.4213/tmf6694

Использование Общероссийского математического портала Math-Net.Ru подразумевает, что вы прочитали и согласны с пользовательским соглашением http://www.mathnet.ru/rus/agreement

Параметры загрузки :

IP : 35.173 .219 .149

26 апреля 2023 г., 13:36:48

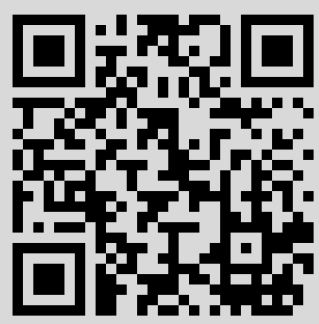




\title{
ФИЗИКА
}

Том 168, № 3

сентябрь, 2011

\section{О КОСВЕННОМ ВЗАИМОДЕЙСТВИИ МЕЖДУ ЯДЕРНЫМИ СПИНАМИ В ЛЕГКООСНОМ АНТИФЕРРОМАГНЕТИКЕ}

\begin{abstract}
Рассматриваются два атома с ядерными спинами $I=1 / 2$, принадлежащие регулярной цепочке (спиновому регистру) из изотопов, замещающих свободные от ядерных спинов базовые атомы в пластине антиферромагнетика с легкой осью анизотропии. Внешнее постоянное магнитное поле предполагается направленным вдоль легкой оси, перпендикулярной плоскости пластины, и обладает постоянным градиентом вдоль направления регистра. Для простой модели, в спин-волновом приближении, с помощью преобразования Боголюбова-Тябликова выполнена диагонализация спинового гамильтониана. Показано, что при наличии неоднородности внешнего магнитного поля косвенное взаимодействие между ядерными спинами, обусловленное сверхтонким взаимодействием ядерных спинов с виртуальными спиновыми волнами в антиферромагнетике, может возрастать и даже осциллировать в зависимости от расстояния между рассматриваемыми спинами, если локальное значение поля в средней точке между ними близко к полю ориентационного фазового перехода в однородном антиферромагнетике в опрокинутую фазу.
\end{abstract}

Ключевые слова: антиферромагнетик с легкой осью анизотропии в неоднородном внешнем поле, преобразование Боголюбова-Тябликова, ориентационный фазовый переход в опрокинутую фазу, косвенное взаимодействие ядерных спинов.

\section{1. ВВЕДЕНИЕ}

Теория косвенного взаимодействия между ядерными спинами в изотропном антиферромагнетике, которое обусловлено сверхтонким взаимодействием ядерных спинов с виртуальными спиновыми волнами при наличии внешнего поля, впервые была сформулирована в работе [1]. Ее изложение можно найти в книгах [2]-[4].

В настоящей статье рассматривается косвенное взаимодействие между ядерными спинами $I=1 / 2$ в простой модели тонкой пластины двухподрешеточного антиферромагнетика с легкой осью анизотропии вдоль внешнего поля, направленного перпендикулярно плоскости пластины. Предполагается, что базисные атомы антиферромагнетика не содержат ядерных спинов. Существенно, что энергетический

*Физико-технологический институт РАН, Москва, Россия. E-mail: aakokin@mail.ru

${ }^{\dagger}$ Институт радиотехники и электроники им. В. А. Котельникова РАН, Москва, Россия 
спектр спиновых волн (магнонов) в этом случае характеризуется энергетической щелью, которая захлопывается при достижении критического значения поля, при этом происходит ориентационный фазовый переход (ОФП) антиферромагнитной фазы в опрокинутую (spin-flop) фазу. Для вычисления косвенного межъядерного взаимодействия вблизи точки ОФП использованы асимптотические по малому параметру, характеризующему величину градиента поля, значения коэффициентов унитарного преобразования Боголюбова-Тябликова спинового гамильтониана антиферромагнитной пластины в спин-волновом приближении.

Настоящая статья представляет собой дальнейшее уточнение и развитие результатов для модели ЯМР-квантового компьютера, полученных нами в работах [5], [6].

\section{2. СПИНОВЫЙ ГАМИЛЬТОНИАН ЛЕГКООСНОЙ АНТИФЕРРОМАГНИТНОЙ ПЛАСТИНЫ В СПИН-ВОЛНОВОМ ПРИБЛИЖЕНИИ}

Рассматриваемая модель антиферромагнитной пластины толщины $d$ представляет собой две эквивалентные, вставленные друг в друга, магнитные тетрагональные подрешетки $\mathbf{A}$ и $\mathbf{B}$. Отдельные узлы подрешеток $\mathbf{A}$ и $\mathbf{B}$ нумеруются соответственно индексами $i$ и $j$. Полное число узлов в каждой подрешетке равно $N=N_{\perp} N_{z}$, где $N_{\perp}=N_{x} N_{y} \gg 1$ - число узлов подрешетки в плоскости пластины $(x, y)$, а $N_{z}=d / a_{z}>1$ - число узлов подрешетки в направлении оси $z$. Период каждой подрешетки в плоскости пластины равен $a_{\perp}$, а в направлении, перпендикулярном плоскости пластины (вдоль легкой оси), период равен $a_{z}$ (см. также [5], [6]). Внешнее постоянное магнитное поле, направленное параллельно легкой оси анизотропии (оси $z$ ), имеет постоянный градиент $d B(x) / d x=G$ вдоль цепочки ядерных спинов (вдоль оси $x$ ), $G \sim 10^{-3}$ Тл/мкм и более.

Исходный спиновый гамильтониан трехмерной модели легкоосного антиферромагнетика, в котором спины одной подрешетки взаимодействуют только с $Z$ ближайшими соседними спинами, принадлежащими другой подрешетке, представим в следующем виде:

$$
\begin{aligned}
\mathbf{H}_{\mathrm{S}}= & \gamma_{\mathrm{S}} \hbar\left(\sum_{i=1}^{N} B\left(x_{i}\right) S_{\mathbf{A}, z}\left(\mathbf{r}_{i}\right)+\sum_{j=1}^{N} B\left(x_{j}\right) S_{\mathbf{B}, z}\left(\mathbf{r}_{i}\right)\right)+ \\
& +\frac{2 \gamma_{\mathrm{S}} \hbar}{Z} \sum_{i=1}^{N} \sum_{\delta=1}^{Z}\left\{B_{\mathrm{E}} \mathbf{S}_{\mathbf{A}}\left(\mathbf{r}_{i}\right) \otimes \mathbf{S}_{\mathbf{B}}\left(\mathbf{r}_{i}+r_{\delta}\right)+B_{\mathrm{A}} S_{\mathbf{A}, z}\left(\mathbf{r}_{i}\right) \otimes S_{\mathbf{B}, z}\left(\mathbf{r}_{i}+r_{\delta}\right)\right\},
\end{aligned}
$$

где $\gamma_{\mathrm{S}} / 2 \pi=28$ ГГц/Тл - гиромагнитное отношение для электронного спина, $\hbar=$ $1.054 \cdot 10^{-34}$ Дж $\cdot$ с, для тетрагональной подрешетки $Z=6, \mathbf{S}_{\mathbf{A}}\left(\mathbf{r}_{i}\right)$ и $\mathbf{S}_{\mathbf{B}}\left(\mathbf{r}_{j}\right)-$ вектор-операторы спинов в узлах, принадлежащих соответственно подрешеткам $\mathbf{A}$ и $\mathbf{B}$ (знак $\otimes$, обозначающий прямое (тензорное) произведение спиновых операторов, далее будем для краткости опускать). Мы будем считать, что $B(x)=B+G x$, где $B$ - значение поля в точке начала отсчета координаты $x$, в которой зеемановская энергия электронных спинов минимальна. Параметры $B_{\mathrm{E}} \sim\left(10 \div 10^{3}\right)$ Тл и $B_{\mathrm{A}} \sim\left(10^{-2} \div 1\right)$ Тл $>0$ суть обменное поле и поле анизотропии антиферромагнетика с легкой осью анизотропии. Составляющие радиус-векторов положения узлов подрешеток, в которых локализованы магнитные атомы, будем выражать через безразмерные величины, при этом сохраняя для них прежние обозначения: $\mathbf{r}_{i}=\left(x_{i}, y_{i}, z_{i}\right) \rightarrow\left(a_{\perp} x_{i}, a_{\perp} y_{i}, a_{z} z_{i}\right)$. Далее $\left(x_{i}, y_{i}\right), z_{i}=\mathbf{r}_{\perp}, z-$ безразмерные координаты радиус-вектора. Элементарный объем обратной подрешетки равен единице. 
Поскольку при больших значениях $N_{x}, N_{y}$ граничные условия для спиновых состояний принято представлять в виде периодических условий Борна-Кармана, удобно перейти от спиновых операторов в узельном представлении к операторам рождения и уничтожения спиновых волн - магнонов, распространяющихся с безразмерными волновыми векторами $\mathbf{q} \equiv\left(\mathbf{q}_{\perp}, q_{z}\right)$.

Мы будем рассматривать антиферромагнитные пластины с такой толщиной $d$, что можно ограничиться учетом только нулевой (однородной вдоль оси $z$ ) моды с $q_{z}=0$. Соответствующее условие обсуждалось нами в работе [6]. В этом случае спиновые волны характеризуются плоским волновым вектором $\mathbf{q}_{\perp} \equiv\left(q_{x}, q_{y}\right)$ с дискретными значениями составляющих, описывающих распространение волн вдоль тонкой пластины. В результате мы приходим к упрощенной квазидвумерной модели для гамильтониана антиферромагнитной пластины.

Учитывая, что при больших значениях $N_{\perp}$ распределение плоских векторов $\mathbf{q}_{\perp}$ приобретает практически непрерывный характер, перейдем от магнонных операторов с дискретными значениями волновых векторов к операторам, зависящим от непрерывных значений $\mathbf{q}_{\perp}$, а от сумм по значениям $\mathbf{q}_{\perp}-$ к интегралам в пределах элементарной ячейки квазидвумерной обратной подрешетки. Спиновые операторы атомов отдельных подрешеток $\mathbf{A}$ и $\mathbf{B}$ в плоской пластине могут быть выражены в волновом представлении следующим образом:

$$
\begin{aligned}
S_{\mathbf{A}, x}\left(\mathbf{r}_{i}\right)-i S_{\mathbf{A}, y}\left(\mathbf{r}_{i}\right)=S_{\mathbf{A}}^{-}\left(\mathbf{r}_{i}\right) & \equiv a_{i}^{+}=\frac{1}{2 \pi} \int a^{+}\left(\mathbf{q}_{\perp}\right) e^{i \mathbf{q}_{\perp} \mathbf{r}_{\perp, i}} d \mathbf{q}_{\perp}, \\
S_{\mathbf{A}, x}\left(\mathbf{r}_{i}\right)+i S_{\mathbf{A}, y}\left(\mathbf{r}_{i}\right)=S_{\mathbf{A}}^{+}\left(\mathbf{r}_{i}\right) \equiv a_{i} & =\frac{1}{2 \pi} \int a^{+}\left(\mathbf{q}_{\perp}\right) e^{-i \mathbf{q}_{\perp} \mathbf{r}_{\perp, i}} d \mathbf{q}_{\perp}, \\
S_{\mathbf{B}}^{-}\left(\mathbf{r}_{j}\right) \equiv b_{j} & =\frac{1}{2 \pi} \int b\left(\mathbf{q}_{\perp}\right) e^{-i \mathbf{q}_{\perp} \mathbf{r}_{\perp, i}} d \mathbf{q}_{\perp} \\
S_{\mathbf{B}}^{+}\left(\mathbf{r}_{j}\right) & \equiv b_{j}^{+}=\frac{1}{2 \pi} \int b^{+}\left(\mathbf{q}_{\perp}\right) e^{i \mathbf{q}_{\perp} \mathbf{r}_{\perp, i}} d \mathbf{q}_{\perp}
\end{aligned}
$$

При этом

$$
S_{\mathbf{A}, z}\left(\mathbf{r}_{i}\right)=\frac{1}{2}-a_{i}^{+} a_{i}, \quad S_{\mathbf{B}, z}\left(\mathbf{r}_{j}\right)=-\frac{1}{2}+b_{j}^{+} b_{j}
$$

Произведения $a_{i}^{+} a_{i}$ и $b_{j}^{+} b_{j}$ спиновых операторов в узельном представлении имеют собственные значения 0 и 1 . При низких температурах $\left\langle 0\left|a_{i}^{+} a_{i}\right| 0\right\rangle \ll 1,\left\langle 0\left|b_{j}^{+} b_{j}\right| 0\right\rangle \ll 1$, и можно считать, что спиновые волны создаются в основном поперечными составляющими спина (спин-волновое приближение). Перестановочные соотношения для этих спиновых операторов приобретают при этом, как известно [7], бозеподобный вид. Если внешнее поле неоднородно вдоль оси $x$, то составляющие вектора $q_{x}$ в отличие от составляющих $q_{y}, q_{z}$ не являются интегралами движения для магнонов. Их состояния имеют вид суперпозиции состояний со значениями волновых векторов, относящимися к разным ячейкам обратной подрешетки $q_{x}$ и $q_{x}^{\prime}=q_{x}+Q_{x}$, где $Q_{x}=2 \pi m_{x}$ есть $x$-составляющая вектора обратной плоской подрешетки, $m_{x}=$ $0, \pm 1, \pm 2, \ldots$. Смещение для составляющих $q_{y}$ на величину $Q_{y}$ является идентичным преобразованием, и поэтому далее мы будем считать, что $\mathbf{Q}_{\perp}=\left(Q_{x}, Q_{y}\right)$ с $Q_{x}=2 \pi m_{x}, Q_{y}=0$. 
Вводя далее безразмерные величины

$$
\omega_{\mathrm{E}}=\gamma_{\mathrm{S}} B_{\mathrm{E}}, \quad 0<b_{\mathrm{A}}=\frac{B_{\mathrm{A}}}{B_{\mathrm{E}}}<1, \quad 0<b=\frac{B}{B_{\mathrm{E}}}, \quad g=\frac{G a_{\perp}}{B_{\mathrm{E}}} \sim 10^{-5}
$$

и используя соотношения (1), (2), получим следующее выражение для гамильтониана рассматриваемой модели с точностью до членов, квадратичных относительно операторов $a\left(\mathbf{q}_{\perp}\right)$ и $b\left(\mathbf{q}_{\perp}\right)$ (спин-волновое приближение):

$$
\begin{aligned}
\mathbf{h}_{\mathrm{S}}= & \frac{\mathbf{H}_{\mathrm{S}}}{\hbar \omega_{\mathrm{E}}}+\frac{N\left(1+b_{\mathrm{A}}\right)}{2}= \\
= & \int \sum_{\mathbf{Q}_{\perp}}\left\{\left(1+b_{\mathrm{A}}-b\right) a^{+}\left(\mathbf{q}_{\perp}+\mathbf{Q}_{\perp}\right) a\left(\mathbf{q}_{\perp}\right)+\left(1+b_{\mathrm{A}}+b\right) b^{+}\left(-\mathbf{q}_{\perp}\right) b\left(-\left(\mathbf{q}_{\perp}+\mathbf{Q}_{\perp}\right)\right)+\right. \\
& +i g\left[a^{+}\left(\mathbf{q}_{\perp}+\mathbf{Q}_{\perp}\right) \frac{\partial a\left(\mathbf{q}_{\perp}\right)}{\partial q_{x}}-\frac{\partial b^{+}\left(-\mathbf{q}_{\perp}\right)}{\partial q_{x}} b\left(-\left(\mathbf{q}_{\perp}+\mathbf{Q}_{\perp}\right)\right)\right]+ \\
& \left.+\gamma_{\mathbf{q}_{\perp}}\left[a^{+}\left(\mathbf{q}_{\perp}+\mathbf{Q}_{\perp}\right) b^{+}\left(-\mathbf{q}_{\perp}\right)+a\left(\mathbf{q}_{\perp}\right) b\left(-\left(\mathbf{q}_{\perp}+\mathbf{Q}_{\perp}\right)\right)\right]\right\} d \mathbf{q}_{\perp},
\end{aligned}
$$

где для модели с простой тетрагональной решеткой $\left(Z=6, \mathbf{r}_{\delta}=( \pm 1 / 2, \pm 1 / 2, \pm 1 / 2)\right)$

$$
\gamma_{\mathbf{q}_{\perp}}=\gamma_{\mathbf{q}_{\perp}}^{*}=\left.\gamma_{\mathbf{q}_{\perp}, q_{z}}\right|_{q_{z}=0}=\frac{\cos \left(q_{x} / 2\right)+\cos \left(q_{y} / 2\right)+1}{3} .
$$

Гамильтониан антиферромагнетика в форме (3) учитывает корреляции магнонных состояний в разных ячейках обратной подрешетки, что выражается суммированием по всем составляющим векторов обратной подрешетки $Q_{x}$.

Переходя далее от спиновых операторов в узельном представлении к магнонным операторам $a\left(\mathbf{q}_{\perp}\right), a^{+}\left(\mathbf{q}_{\perp}^{\prime}\right)$ и $b\left(\mathbf{q}_{\perp}\right), b^{+}\left(\mathbf{q}_{\perp}^{\prime}\right)$ с непрерывными аргументами, получим (остальные коммутаторы равны нулю)

$$
\begin{aligned}
{\left.\left[a\left(\mathbf{q}_{\perp}\right), a^{+}\left(\mathbf{q}_{\perp}^{\prime}\right)+\mathbf{Q}_{\perp}^{\prime}\right)\right] } & =\sum_{\mathbf{Q}_{\perp} \delta\left(\mathbf{q}_{\perp}^{\prime}-\mathbf{q}_{\perp}-\mathbf{Q}_{\perp}+\mathbf{Q}_{\perp}^{\prime}\right)} \\
{\left[b^{+}\left(-\mathbf{q}_{\perp}\right), b\left(-\left(\mathbf{q}_{\perp}^{\prime}+\mathbf{Q}_{\perp}^{\prime}\right)\right)\right] } & =-\sum_{\mathbf{Q}_{\perp}} \delta\left(\mathbf{q}_{\perp}^{\prime}-\mathbf{q}_{\perp}-\mathbf{Q}_{\perp}+\mathbf{Q}_{\perp}^{\prime}\right) .
\end{aligned}
$$

Для магнонных операторов $a(\tau, \mathbf{q})$ и $b^{+}(\tau,-\mathbf{q})$ в представлении Гейзенберга (здесь и далее $\tau=\omega_{\mathrm{E}} t-$ безразмерный временной аргумент), используя гамильтониан (3), правила коммутации (4) и вводя обозначение $\mathbf{Q}_{\perp}-\mathbf{Q}_{\perp}^{\prime}=\mathbf{Q}_{\perp}^{\prime \prime}$, получим линейную систему уравнений движения:

$$
\begin{aligned}
& i \frac{\partial a\left(\tau, \mathbf{q}_{\perp}\right)}{\partial \tau}=\left[a\left(\tau, \mathbf{q}_{\perp}\right), \mathbf{h}_{\mathrm{S}}\right]= \\
& =\sum_{\mathbf{Q}_{\perp}^{\prime \prime}}\left\{\left(1+b_{\mathrm{A}}-b-i g \frac{\partial}{\partial q_{x}}\right) a\left(\tau, \mathbf{q}_{\perp}+\mathbf{Q}_{\perp}^{\prime \prime}\right)+\gamma_{\mathbf{q}_{\perp}} b^{+}\left(\tau,-\left(\mathbf{q}_{\perp}+\mathbf{Q}_{\perp}^{\prime \prime}\right)\right)\right\}, \\
& i \frac{\partial b^{+}\left(\tau,-\mathbf{q}_{\perp}\right)}{\partial \tau}=\left[b^{+}\left(\tau,-\mathbf{q}_{\perp}\right), \mathbf{h}_{\mathrm{S}}\right]= \\
& =-\sum_{\mathbf{Q}_{\perp}^{\prime \prime}}\left\{\left(1+b_{\mathrm{A}}+b+i g \frac{\partial}{\partial q_{x}}\right) b^{+}\left(\tau,-\left(\mathbf{q}_{\perp}+\mathbf{Q}_{\perp}^{\prime \prime}\right)\right)+\gamma_{\mathbf{q}_{\perp}} a\left(\tau, \mathbf{q}_{\perp}+\mathbf{Q}_{\perp}^{\prime \prime}\right)\right\} .
\end{aligned}
$$


Видно, что учет процессов переброса состояний в другие элементарные ячейки обратной решетки приводит к системе зацепляющихся уравнений движения для магнонных операторов из различных ячеек обратной подрешетки.

Далее в перестановочных соотношениях (4), учитывая малость градиента внешнего поля, будем пренебрегать процессами переброса между разными ячейками обратной подрешетки, т. е. положим $\mathbf{Q}_{\perp}=\mathbf{Q}_{\perp}^{\prime}=0$ (назовем это приближение $\mathrm{N}$-приближением).

\section{3. ДИАГОНАЛИЗАЦИЯ СПИНОВОГО ГАМИЛЬТОНИАНА АНТИФЕРРОМАГНЕТИКА}

Перейдем теперь к новым линейно независимым операторам рождения и уничтожения $\xi^{+}\left(\tau, q_{y}, E_{ \pm}\right)$и $\xi\left(\tau, q_{y}, E_{ \pm}^{\prime}\right)$ магнонов двух типов, распространяющихся с безразмерными энергиями $E_{ \pm}=E \pm b$, где $E$ - непрерывный параметр, и составляющей волнового вектора $q_{y}$, лежащей в интервале от $q_{y}$ до $q_{y}+d q_{y}$.

Из четырех уравнений преобразования выпишем здесь только два уравнения (два других уравнения являются эрмитово сопряженными)

$$
\begin{aligned}
a\left(\tau, \mathbf{q}_{\perp}\right) & =\int\left(u\left(\mathbf{q}_{\perp}, E\right) \xi\left(\tau, q_{y}, E_{-}\right)+v^{*}\left(\mathbf{q}_{\perp}, E\right) \xi^{+}\left(\tau, q_{y}, E_{+}\right)\right) d E, \\
b^{+}\left(\tau,-\mathbf{q}_{\perp}\right) & =\int\left(v\left(\mathbf{q}_{\perp}, E\right) \xi\left(\tau, q_{y}, E_{-}\right)+u^{*}\left(\mathbf{q}_{\perp}, E\right) \xi^{+}\left(\tau, q_{y}, E_{+}\right)\right) d E .
\end{aligned}
$$

Это преобразование можно рассматривать как обобщение преобразования Боголюбова-Тябликова [7] на случай легкоосного антиферромагнетика с неоднородным вдоль оси $x$ внешним полем.

Введенные операторы будут удовлетворять перестановочным соотношениям

$$
\begin{aligned}
& {\left[\xi\left(\tau, q_{y}, E_{ \pm}\right), \xi^{+}\left(\tau, q_{y}^{\prime}, E_{ \pm}^{\prime}\right)\right]=\delta\left(q_{y}-q_{y}^{\prime}\right) \delta\left(E-E^{\prime}\right),} \\
& {\left[\xi\left(\tau, q_{y}, E_{ \pm}\right), \xi\left(\tau, q_{y}^{\prime}, E_{ \pm}^{\prime}\right)\right]=\left[\xi^{+}\left(\tau, q_{y}, E_{ \pm}\right), \xi^{+}\left(\tau, q_{y}^{\prime}, E_{ \pm}^{\prime}\right)\right]=} \\
& \quad=\left[\xi\left(\tau, q_{y}, E_{ \pm}\right), \xi\left(\tau, q_{y}^{\prime}, E_{\mp}^{\prime}\right)\right]=\left[\xi^{+}\left(\tau, q_{y}, E_{ \pm}\right), \xi^{+}\left(\tau, q_{y}^{\prime}, E_{\mp}^{\prime}\right)\right]=0,
\end{aligned}
$$

если, как это следует из записанных в $\mathrm{N}$-приближении соотношений (4), выполняются следующие два условия унитарности для коэффициентов преобразования:

$$
\begin{aligned}
& \int\left(u\left(\mathbf{q}_{\perp}, E\right) u^{*}\left(q_{x}^{\prime}, q_{y}, E\right)-v^{*}\left(\mathbf{q}_{\perp}, E\right) v\left(q_{x}^{\prime}, q_{y}, E\right)\right) d E=\delta\left(q_{x}-q_{x}^{\prime}\right), \\
& \int\left(v^{*}\left(\mathbf{q}_{\perp}, E\right) u\left(q_{x}^{\prime}, q_{y}, E\right)-u\left(\mathbf{q}_{\perp}, E\right) v^{*}\left(q_{x}^{\prime}, q_{y}, E\right)\right) d E=0 .
\end{aligned}
$$

Найдем теперь условия, при которых новые операторы будут также удовлетворять уравнениям движения

$$
\begin{gathered}
i \frac{\partial \xi\left(\tau, q_{y}, E_{ \pm}\right)}{\partial \tau}=\left[\xi\left(\tau, q_{y}, E_{ \pm}\right), \mathbf{h}_{\mathrm{S}}(\tau)\right]=E_{ \pm} \xi\left(\tau, q_{y}, E_{ \pm}\right) \\
i \frac{\partial \xi^{+}\left(\tau, q_{y}, E_{ \pm}\right)}{\partial \tau}=\left[\xi^{+}\left(\tau, q_{y}, E_{ \pm}\right), \mathbf{h}_{\mathrm{S}}(\tau)\right]=-E_{ \pm} \xi^{+}\left(\tau, q_{y}, E_{ \pm}\right) .
\end{gathered}
$$

Для этого подставим соотношения (6) и (9) в уравнения движения (5), записанные в N-приближении, и приравняем нулю в полученных подынтегральных выражениях коэффициенты при операторах $\xi\left(\tau, q_{y}, E_{-}\right)$и $\xi^{+}\left(\tau, q_{y}, E_{+}\right)$. В результате для 
определения коэффициентов преобразования $u\left(\mathbf{q}_{\perp}, E\right), v\left(\mathbf{q}_{\perp}, E\right)$ имеем следующие уравнения:

$$
\begin{aligned}
\left(i g \frac{\partial}{\partial q_{x}}-\left(E-1-b_{\mathrm{A}}\right)\right) u\left(\mathbf{q}_{\perp}, E\right)+\gamma_{\mathbf{q}_{\perp}} v\left(\mathbf{q}_{\perp}, E\right) & =0, \\
\gamma_{\mathbf{q}_{\perp}} u\left(\mathbf{q}_{\perp}, E\right)-\left(i g \frac{\partial}{\partial q_{x}}-\left(E+1+b_{\mathrm{A}}\right)\right) v\left(\mathbf{q}_{\perp}, E\right) & =0, \\
\left(i g \frac{\partial}{\partial q_{x}}+\left(E+1+b_{\mathrm{A}}\right)\right) v^{*}\left(\mathbf{q}_{\perp}, E\right)+\gamma_{\mathbf{q}_{\perp}} u^{*}\left(\mathbf{q}_{\perp}, E\right) & =0, \\
\gamma_{\mathbf{q}_{\perp}} v^{*}\left(\mathbf{q}_{\perp}, E\right)-\left(i g \frac{\partial}{\partial q_{x}}+\left(E-1-b_{\mathrm{A}}\right)\right) u^{*}\left(\mathbf{q}_{\perp}, E\right) & =0 .
\end{aligned}
$$

Заметим, что первая пара уравнений переходит во вторую пару, если сделать замены $u\left(\mathbf{q}_{\perp}, E\right) \rightarrow v^{*}\left(\mathbf{q}_{\perp},-E\right)$ и $v\left(\mathbf{q}_{\perp}, E\right) \rightarrow u^{*}\left(\mathbf{q}_{\perp},-E\right)$. При этом, однако, условия (8) не переходят сами в себя. Это означает, что решения со значениями $E<0$ являются нефизическими, и их следует исключить (см. также книгу [7]). Если $E>0$, то коэффициенты преобразования в обеих парах уравнений отличаются только комплексным сопряжением, поэтому далее мы будем пользоваться только одной (второй) парой уравнений (10).

Запишем выражение для гамильтониана (3) в другой форме. Умножим первое уравнение в $(5)$, записанное в $\mathrm{N}$-приближении, слева на $a^{+}\left(\tau, q_{x}+Q_{x}, q_{y}\right)$, второе уравнение в $(5)$ - справа на $b\left(\tau,-\left(q_{x}+Q_{x}\right),-q_{y}\right)$, а разность этих выражений просуммируем по переменной $Q_{x}$ и проинтегрируем по $\mathbf{q}_{\perp}$. Видно, что правая часть полученного выражения совпадает с (3). В результате имеем

$$
\begin{aligned}
\mathbf{h}_{\mathrm{S}}=\mathbf{h}_{\mathrm{S}}(\tau)= & \int \sum_{Q_{x}}\left(i a^{+}\left(\tau, q_{x}+Q_{x}, q_{y}\right) \frac{\partial a\left(\tau, \mathbf{q}_{\perp}\right)}{\partial \tau}-\right. \\
& \left.-i \frac{\partial b^{+}\left(\tau,-\mathbf{q}_{\perp}\right)}{\partial \tau} b\left(\tau,-\left(q_{x}+Q_{x},-q_{y}\right)\right)\right) d \mathbf{q}_{\perp} .
\end{aligned}
$$

Следующее условие должно обеспечивать диагонализацию полученных при подстановке в выражение (11) соотношений (7) и (9). Этого можно достичь, если потребовать обращения в ноль слагаемых, содержащих произведение магнонных операторов типа $\xi\left(\tau, q_{y}, E_{+}\right) \xi\left(\tau, q_{y}, E_{-}^{\prime}\right)$ и $\xi^{+}\left(\tau, q_{y}, E_{+}\right) \xi^{+}\left(\tau, q_{y}, E_{-}^{\prime}\right)$, т. е. потребовать выполнения равенства

$$
\sum_{\mathbf{Q}_{\perp}} \int\left(v\left(\mathbf{q}_{\perp}+\mathbf{Q}_{\perp}, \mathbf{q}_{\perp}, E\right) u\left(\mathbf{q}_{\perp}, E^{\prime}\right)-v\left(\mathbf{q}_{\perp}, E^{\prime}\right) u\left(\mathbf{q}_{\perp}+\mathbf{Q}_{\perp}, E\right)\right) d \mathbf{q}_{\perp}=0
$$

и его комплексно-сопряженного аналога. Наконец, должно иметь место условие нормировки

$$
\sum_{\mathbf{Q}_{\perp}} \int\left(u^{*}\left(\mathbf{q}_{\perp}+\mathbf{Q}_{\perp}, E^{\prime}\right) u\left(\mathbf{q}_{\perp}, E\right)-v^{*}\left(\mathbf{q}_{\perp}+\mathbf{Q}_{\perp}, E^{\prime}\right) v\left(\mathbf{q}_{\perp}, E\right)\right) d \mathbf{q}_{\perp}=\delta\left(E-E^{\prime}\right) .
$$

Вместе с первым перестановочным соотношением из системы (7) и с учетом соотношений

$$
\lim _{q_{y} \rightarrow q^{\prime}} \delta\left(q_{y}-q_{y}^{\prime}\right)=\frac{N_{y}}{2 \pi}, \quad\left[\xi\left(\tau, q_{y}, E_{ \pm}\right), \xi^{+}\left(\tau, q_{y}, E_{ \pm}^{\prime}\right)\right]=\frac{N_{y}}{2 \pi} \delta\left(E-E^{\prime}\right)
$$


оно позволяет получить для спинового гамильтониана диагонализованный вид:

$$
\begin{aligned}
& \mathbf{h}_{\mathrm{S}}(\tau)=\frac{N_{y}}{2 \pi} \int \sum_{Q_{x}}\left(E_{+} v^{*}\left(\mathbf{q}_{\perp}, E\right) v\left(q_{x}+Q_{x}, q_{y}, E\right)+\right. \\
& \left.\quad+E_{-} v^{*}\left(q_{x}+Q_{x}, q_{y}, E\right) v\left(\mathbf{q}_{\perp}, E\right)\right) d \mathbf{q}_{\perp} d E+ \\
& \quad+\int\left(E_{+} \xi^{+}\left(\tau, q_{y}, E_{+}\right) \xi\left(\tau, q_{y}, E_{+}\right)+E_{-} \xi^{+}\left(\tau, q_{y}, E_{-}\right) \xi\left(\tau, q_{y}, E_{-}\right)\right) d q_{y} d E .
\end{aligned}
$$

Последнее слагаемое в данном выражении описывает энергию магнонных возбуждений и согласуется с принятыми выше уравнениями движения для магнонных операторов (9). Достаточным условием того, что квадратичная форма (13) описывает устойчивое состояние системы, близкое к основному, является положительность энергии обоих типов магнонов: $E_{ \pm}=E \pm b>0$.

\section{4. АСИМПТОТИЧЕСКОЕ РЕШЕНИЕ УРАВНЕНИЯ ДЛЯ КОЭФФИЦИЕНТОВ ПРЕОБРАЗОВАНИЯ}

Перейдем теперь к вычислению коэффициентов преобразования. Воспользуемся второй парой уравнений (10). Исключим величину $v^{*}\left(\mathbf{q}_{\perp}, E\right)$ и заменим переменную $q_{x}$ на $\zeta=\int_{0}^{q_{x}} \gamma_{\mathbf{q}_{\perp}} d q_{x}$. Поскольку переменная $q_{y}$ в уравнениях (10) рассматривается как параметр, не будем ее явно указывать в аргументах и перейдем от $u^{*}\left(\mathbf{q}_{\perp}, E\right)$ и $\gamma_{\mathbf{q}_{\perp}}$ к обозначениям $u^{*}(\zeta, E)$ и $\gamma_{\zeta}$, а частные производные по $\zeta$ заменим на обыкновенные. В результате для определения коэффициента преобразования $u^{*}(\zeta, E)$ получим дифференциальное уравнение с малым параметром $g \ll 1$ :

$$
g^{2} \frac{d^{2} u^{*}(\zeta, E)}{d \zeta^{2}}+p(\zeta, g) g \frac{d u^{*}(\zeta, E)}{d \zeta}+r(\zeta, g) u^{*}(\zeta, E)=0,
$$

где введены обозначения

$$
\begin{aligned}
& p(\zeta, g)=p_{0}(\zeta)=-2 i \frac{E}{\gamma_{\zeta}}, \\
& r(\zeta, g)=r_{0}(\zeta)+g r_{1}(\zeta)=\frac{\left(1+b_{\mathrm{A}}\right)^{2}-E^{2}-\gamma_{\zeta}^{2}}{\gamma_{\zeta}^{2}}+i g\left(1+b_{\mathrm{A}}-E\right) \frac{d\left(1 / \gamma_{\zeta}\right)}{d \zeta}
\end{aligned}
$$

Решение уравнения (14) будем искать в виде асимптотического разложения по малому параметру $g \ll 1[8]$ :

$$
u^{*}(\zeta, E)=\sum_{n=0}^{\infty} g^{n}\left\{A_{n}(\zeta) e^{\theta_{+}(\zeta)}+B_{n}(\zeta) e^{\theta_{-}(\zeta)}\right\}, \quad \frac{d \theta_{ \pm}}{d \zeta}=-\frac{\lambda_{ \pm}(\zeta)}{g}
$$

где величины $\theta_{ \pm}$и $\zeta$ предполагаются независимыми, а значения $\lambda_{ \pm}(\zeta)$ определяются ниже. После подстановки в уравнение (14) данного разложения приравниваем к нулю выражения при $g^{n} e^{\theta_{ \pm}}$и, ограничиваясь далее первым порядком малости по параметру $g$ (т. е. рассматривая слагаемые с $n=0,1)$, получим

$$
\lambda_{ \pm}=\frac{i}{\gamma_{\zeta}}(-E \pm E(\zeta)), \quad E(\zeta)=\sqrt{\left(1+b_{\mathrm{A}}\right)^{2}-\gamma_{\zeta}^{2}}
$$


Для определения величин $A_{0}(\zeta), B_{0}(\zeta)$ мы имеем два дифференциальных уравнения (здесь штрих обозначает производную по $\zeta$ )

$$
\begin{aligned}
& \left(2 \lambda_{+}+p_{0}\right) A_{0}^{\prime}+\left(\lambda_{+}^{\prime}+r_{+}\right) A_{0}=0 \\
& \left(2 \lambda_{-}+p_{0}\right) B_{0}^{\prime}+\left(\lambda_{-}^{\prime}+r_{-}\right) B_{0}=0
\end{aligned}
$$

решения которых представим в виде

$$
A_{0}(\zeta) e^{\theta_{+}}=A e^{I_{+}(\zeta)}, \quad B_{0}(\zeta) e^{\theta_{-}}=B e^{I_{-}(\zeta)},
$$

где

$$
I_{ \pm}(\zeta)=-\frac{1}{2}\left(\ln \frac{E(\zeta) / \gamma_{\zeta}}{E(0) / \gamma_{0}} \pm\left(\frac{E(\zeta)}{\gamma_{\zeta}}-\frac{E(0)}{\gamma_{0}}\right)\right)-\frac{1}{g} \int_{0}^{\zeta} \lambda_{ \pm} d \zeta
$$

а постоянные интегрирования $A=A\left(E, q_{y}\right), B=B\left(E, q_{y}\right)$ могут зависеть от $E$ и $q_{y}$ как от параметров.

Поскольку $E>0$, величина $\lambda_{+}$может принимать сколь угодно малые значения. В пределе $g \rightarrow 0$ мы имеем $\lambda_{+} \rightarrow 0$, что совпадает с известным результатом для двух ветвей магнонного спектра в случае однородного поля:

$$
E_{ \pm}=E \pm b \rightarrow E_{ \pm}\left(\mathbf{q}_{\perp}, b\right)=E(\zeta) \pm b=\sqrt{\left(1+b_{\mathrm{A}}\right)^{2}-\gamma_{\zeta}^{2}} \pm b>0
$$

(не следует путать здесь знак \pm с индексом \pm в $I_{ \pm}(\zeta)$ ). Из двух приближенных решений $(15)$ нужно выбирать решение $u^{*}(\zeta, E) \approx A_{0}(\zeta) e^{\theta_{+}}=A e^{I_{+}(\zeta)}$, для которого нижняя ветвь магнонного спектра $E_{-}=E(\zeta)-b>0$. Эта ветвь имеет энергетическую щель, которая исчезает, когда внешнее поле достигает критического значения $b=b_{\mathrm{c}}$, где $b_{\mathrm{c}}=\left.E\left(\mathbf{q}_{\perp}\right)\right|_{\mathbf{q}_{\perp}=0}=\sqrt{\left(1+b_{\mathrm{A}}\right)^{2}-1}=\sqrt{2 b_{\mathrm{A}}+b_{\mathrm{A}}^{2}}$, и в однородном антиферромагнетике происходит ОФП типа опрокидывания (см. дополнение к книге [7] и книгу [9]).

Приближенное выражение для $I_{+}\left(\mathbf{q}_{\perp}, E\right)$ получим, производя с переменной $q_{x}$ преобразование растяжения $q_{x} / g=\eta[8]$. В пределе $g \rightarrow 0$ "растянутая" переменная принимает значения $0<|\eta|<\infty$, при этом

$$
I_{1}\left(\mathbf{q}_{\perp}, E\right)=I_{1}\left(g \eta, q_{y}, E\right)=-i\left(E \eta-\int_{0}^{g \eta} E\left(g \eta, q_{y}\right) d \eta\right)
$$

для любого конечного значения $\eta$. Возвращаясь к исходной переменной и используя теперь два последних уравнения из системы (10), получим следующие асимптотические выражения для коэффициентов преобразования, полагая при этом, что в предэкспоненциальном множителе $E\left(\mathbf{q}_{\perp}\right) \approx E$ в пределе $g \rightarrow 0$ :

$$
\begin{aligned}
& u^{*}\left(\mathbf{q}_{\perp}, E\right) \approx A \exp \left\{\frac{i}{g}\left(E q_{x}-\int_{0}^{q_{x}} E\left(\mathbf{q}_{\perp}\right) d q_{x}\right)\right\}+O(g), \\
& v^{*}\left(\mathbf{q}_{\perp}, E\right) \approx \frac{E-\sqrt{1+b_{\mathbf{c}}^{2}}}{\gamma_{\mathbf{q}_{\perp}}} A \exp \left\{\frac{i}{g}\left(E q_{x}-\int_{0}^{q_{x}} E\left(\mathbf{q}_{\perp}\right) d q_{x}\right)\right\}+O(g) .
\end{aligned}
$$


Для определения коэффициента $A$ подставим данные выражения в нормировочное условие $(12)$ и будем иметь (символ $O(g)$ далее писать не будем)

$$
\begin{aligned}
\frac{2 E A^{2}}{\sqrt{1+b_{\mathrm{c}}^{2}}+E} & \int_{-\infty}^{\infty} \exp \left\{-\frac{i}{g}\left(E-E^{\prime}\right) q_{x}\right\} \times \\
& \times \sum_{m} \exp \left\{-\frac{i}{g}\left(\int_{q_{x}}^{q_{x}+2 \pi m} E\left(\mathbf{q}_{\perp}\right) d q_{x}-2 \pi m E^{\prime}\right)\right\} d q_{x}=\delta\left(E-E^{\prime}\right) .
\end{aligned}
$$

Опустим в этом выражении быстроосциллирующие слагаемые с $m \neq 0$. Окончательно, для обоих коэффициентов унитарного преобразования мы получаем следующие выражения:

$$
\begin{aligned}
& u^{*}\left(\mathbf{q}_{\perp}, E\right)=\frac{1}{\sqrt{4 \pi g}}\left(\frac{\sqrt{1+b_{\mathbf{c}}^{2}}}{E}+1\right)^{1 / 2} \exp \left\{\frac{i}{g}\left(E q_{x}-\int_{0}^{q_{x}} E\left(\mathbf{q}_{\perp}\right) d q_{x}\right)\right\}, \\
& v^{*}\left(\mathbf{q}_{\perp}, E\right)=\frac{1}{\sqrt{4 \pi g}}\left(\frac{\sqrt{1+b_{\mathbf{c}}^{2}}}{E}-1\right)^{1 / 2} \exp \left\{\frac{i}{g}\left(E q_{x}-\int_{0}^{q_{x}} E\left(\mathbf{q}_{\perp}\right) d q_{x}\right)\right\} .
\end{aligned}
$$

Переходя теперь в первом слагаемом в правой части гамильтониана (13) к переменной интегрирования $\varepsilon=E-E\left(\mathbf{q}_{\perp}\right)$ и расширяя пределы интегрирования по этой переменной до $\pm \infty$, получим

$$
\begin{gathered}
\sum_{Q_{x}} \int\left\{E_{+}\left(v^{*}\left(\mathbf{q}_{\perp}, E\right) v\left(\mathbf{q}_{\perp}+\mathbf{Q}_{\perp}, E\right)\right)+E_{-}\left(v\left(\mathbf{q}_{\perp}, E\right) v^{*}\left(\mathbf{q}_{\perp}+\mathbf{Q}_{\perp}, E\right)\right)\right\} d E \approx \\
\approx\left(\sqrt{1+b_{\mathrm{c}}^{2}}-E\left(\mathbf{q}_{\perp}\right)\right) \delta\left(Q_{x}\right)=\left(\sqrt{1+b_{\mathrm{c}}^{2}}-E\left(\mathbf{q}_{\perp}\right)\right) \frac{N_{x}}{2 \pi},
\end{gathered}
$$

где учтено, что $\delta\left(Q_{x}\right) \approx N_{x} / 2 \pi$. В результате выражение для диагонализованного гамильтониана (13) переходит в окончательное приближенное асимптотическое выражение:

$$
\begin{aligned}
\mathbf{h}_{\mathrm{S}} \approx & -\left(1+b_{\mathrm{A}}\right) N_{\perp}+\frac{N_{\perp}}{(2 \pi)^{2}} \int E\left(\mathbf{q}_{\perp}\right) d \mathbf{q}_{\perp}+ \\
& +\int\left\{E_{+} \xi^{+}\left(\tau, q_{y}, E_{+}\right) \xi\left(\tau, q_{y}, E_{+}\right)+E_{-} \xi^{+}\left(\tau, q_{y}, E_{-}\right) \xi\left(\tau, q_{y}, E_{-}\right)\right\} d E d q_{y} .
\end{aligned}
$$

Первые два слагаемых в правой части данного выражения описывают энергию основного состояния антиферромагнетика, включая энергию нулевых колебаний, а последнее слагаемое соответствует динамической части спинового гамильтониана с малыми магнонными возбуждениями.

\section{5. КОСВЕННОЕ ВЗАИМОДЕЙСТВИЕ МЕЖДУ ЯДЕРНЫМИ СПИНАМИ ВБЛИЗИ ТОЧКИ ОФП ТИПА ОПРОКИДЫВАНИЯ}

Пусть атомы $k$ и $l$ в одномерном спиновом регистре на ядерных спинах с $I=1 / 2$ находятся в плоскости пластины в точках с координатами $y_{l, k}=z_{l, k}=0$ и $x_{l, k}$, причем расстояние $x_{l}-x_{k} \equiv l-k>0$. Ограничимся рассмотрением простого случая, когда эти атомы принадлежат одной и той же подрешетке антиферромагнетика $\mathbf{A}$. 
Сверхтонкое взаимодействие ядерного спина атома, имеющего координату $x=k$, с собственным электронным спином будем описывать гамильтонианом, заданным соотношением

$$
\begin{aligned}
& \frac{\mathbf{H}_{\mathrm{IS}}(k)}{\hbar \omega_{\mathrm{E}}}=\mathbf{h}_{\mathrm{IS}}(k)=-\omega_{\mathrm{I}}(k) I_{\mathbf{A}, z}(k)+a \mathbf{I}_{\mathbf{A}}(k) \mathbf{S}_{\mathbf{A}}(k) \approx \\
& \approx-\left(\omega_{\mathrm{I}}(k)-a S_{\mathbf{A}, z}(k)\right) I_{\mathbf{A}, z}(k)+\frac{a}{2}\left(I_{\mathbf{A}}^{+}(k) S_{\mathbf{A}}^{-}(k)+I_{\mathbf{A}}^{-}(k) S_{\mathbf{A}}^{+}(k)\right),
\end{aligned}
$$

где $I_{\mathbf{A}, z}$ и $I_{\mathbf{A}}^{ \pm}=I_{\mathbf{A}, x} \pm i I_{\mathbf{A}, y}-$ составляющие операторов ядерных спинов, $\omega_{\mathrm{I}}(k)=$ $\left(\gamma_{\mathrm{I}} / \gamma_{\mathrm{S}}\right) b_{k}$ - локальная резонансная частота ядерного спина в поле $b_{k} \equiv b+g k$, $\gamma_{\mathrm{I}} / \gamma_{\mathrm{S}} \sim 10^{-3}, a=A / \omega_{\mathrm{E}} \sim 10^{-3}$ - изотропная безразмерная постоянная сверхтонкого взаимодействия $(A / 2 \pi \sim 100$ МГц).

Перейдем теперь в (17) к представлению взаимодействия и сохраним в гамильтониане только линейную зависимость от электронных спиновых операторов:

$$
\mathbf{h}_{\mathrm{IS}}(\tau, k)=-\left(\omega_{\mathrm{I}}(k)-\frac{a}{2}\right) I_{\mathbf{A}, z}(k)+\Delta \mathbf{h}_{\mathrm{IS}}(\tau, k),
$$

где $\Delta \mathbf{h}_{\mathrm{IS}}(\tau, k)$ - гамильтониан рассматриваемого как возмущение "поперечного" сверхтонкого взаимодействия ядерного спина с электронным спином того же $k$-го атома. В представлении взаимодействия он имеет вид

$$
\Delta \mathbf{h}_{\mathrm{IS}}(\tau, k)=e^{i \mathbf{h}_{\mathrm{S}} \tau} \Delta \mathbf{h}_{\mathrm{IS}}(k) e^{-i \mathbf{h}_{\mathrm{S}} \tau}=\frac{a}{2}\left\{I_{\mathbf{A}}^{+}(k) S_{\mathbf{A}}^{-}(\tau, k)+I_{\mathbf{A}}^{-}(k) S_{\mathbf{A}}^{+}(\tau, k)\right\} .
$$

Для рассматриваемой квазидвухмерной антиферромагнитной структуры с учетом (1) и (6) (далее индекс $A$ будем опускать) запишем

$$
\begin{aligned}
S_{\mathbf{A}}^{-}(\tau, k) & =\left(S_{\mathbf{A}}^{+}(\tau, k)\right)^{+}=\frac{1}{2 \pi} \int a^{+}\left(\tau, \mathbf{q}_{\perp}\right) e^{i q_{x} k} d \mathbf{q}_{\perp}= \\
& =\frac{1}{2 \pi} \int\left\{u^{*}\left(\mathbf{q}_{\perp}, E\right) e^{i E_{-} \tau} \xi^{+}\left(q_{y}, E_{-}\right)+v\left(\mathbf{q}_{\perp}, E\right) e^{-i E_{+} \tau} \xi\left(q_{y}, E_{+}\right)\right\} e^{i q_{x} k} d E d \mathbf{q}_{\perp} .
\end{aligned}
$$

Далее учтем, что для невозмущенной матрицы плотности в пределе низких температур

$$
\rho_{\mathrm{S}}(-\infty)=|0\rangle\langle 0|, \quad\left\langle 0\left|S_{\mathbf{A}}^{ \pm}(\tau, j), S_{\mathbf{A}}^{ \pm}\left(\tau+\tau^{\prime}, j^{\prime}\right)\right| 0\right\rangle=0,
$$

и для поправки к гамильтониану двух ядерных спинов во втором порядке теории возмущений получим

$$
\begin{aligned}
& \mathbf{h}_{\mathrm{II}}(k, l)=\operatorname{Tr}_{\mathrm{S}} \rho_{\mathrm{S}}(\tau)\left(\Delta \mathbf{h}_{\mathrm{IS}}(\tau, k)+\Delta \mathbf{h}_{\mathrm{IS}}(\tau, l)\right)= \\
& =-\frac{i a^{2}}{4} \lim _{s \rightarrow+0} \sum_{j, j^{\prime}=k, l} \int_{-\infty}^{0}\left\langle 0\left|\left[I_{\mathbf{A}}^{+}(j) S_{\mathbf{A}}^{-}(\tau, j), I_{\mathbf{A}}^{-}\left(j^{\prime}\right) S_{\mathbf{A}}^{+}\left(\tau+\tau^{\prime}, j^{\prime}\right)\right]\right| 0\right\rangle e^{s \tau^{\prime}} d \tau^{\prime}+\text { э. c. }
\end{aligned}
$$

где $s$ - малый параметр, характеризующий здесь скорость включения сверхтонкого взаимодействия $\left(s \ll b_{\mathrm{c}}-b\right)$. После достаточно громоздких преобразований выражения (18) (см. приложение) для гамильтониана косвенного взаимодействия между двумя ядерными спинами получим (далее индекс А будем опускать)

$$
\Delta \mathbf{h}_{\mathrm{II}}(k, l)=-U(k, l)\left(I^{-}(k) I^{+}(l)+I^{+}(k) I^{-}(l)\right),
$$


где

$$
\begin{aligned}
U(k, l)= & \frac{a^{2}}{2(2 \pi)^{2}} \operatorname{Re} \int\left(\frac{u^{*}\left(q_{x}, q_{y}, E\right) u\left(q_{x}^{\prime}, q_{y}, E\right)}{E_{-}+i s}-\frac{v\left(q_{x}, q_{y}, E\right) v^{*}\left(q_{x}^{\prime}, q_{y}, E\right)}{E_{+}-i s}\right) \times \\
& \times e^{i\left(q_{x} k-q_{x}^{\prime} l\right)} d E d \mathbf{q}_{\perp} d q_{x}^{\prime} .
\end{aligned}
$$

Сохраним далее в этом выражении только основные члены, содержащие в знаменателе $E_{-}=E-b$ (энергию низшей магнонной моды), и произведем следующую замену переменных интегрирования: $\left(q_{x}, q_{x}^{\prime}\right) \rightarrow\left(q_{-}, q_{+}\right)$, где

$$
q_{-}=q_{x}-q_{x}^{\prime}, \quad q_{+}=\frac{q_{x}+q_{x}^{\prime}}{2}, \quad q_{x} k-q_{x}^{\prime} l=q_{-} \frac{l+k}{2}-q_{+}(l-k),
$$

при этом $d q_{x} d q_{x}^{\prime}=d q_{-} d q_{+}$. Воспользуемся опять переходом в экспоненте к растянутой переменной $w=q_{-} / g$. Переход к пределу $g \rightarrow 0$ в предэкспоненциальных множителях, $\left|q_{-}\right|=\left|q_{x}-q_{x}^{\prime}\right|=|w| g \rightarrow 0$, позволяет произвести замены $q_{+}=q_{x}-q_{-} / 2 \rightarrow$ $q_{x}, q_{x}^{\prime}=q_{x}-q_{-} \rightarrow q_{x}$ и $d q_{+} \rightarrow d q_{x}$. В результате из выражения (19) будем иметь

$$
\begin{aligned}
U(k, l)= & \frac{a^{2}}{2(2 \pi)^{2}} \operatorname{Re} \int g \frac{\left|u\left(\mathbf{q}_{\perp}, E\right)\right|^{2}}{E_{-}+i s} \times \\
& \times \exp \left\{-i\left(E-E\left(\mathbf{q}_{\perp}\right)+g \frac{l+k}{2}\right) w-i q_{x}(l-k)\right\} d E d w d \mathbf{q}_{\perp} .
\end{aligned}
$$

Отметим, что знаменатель $E_{-}=E-b$ в (20) может быть мал, но он должен быть больше некоторого минимального значения, как это требуется для применимости теории возмущения второго порядка.

Расширяя пределы интегрирования по переменной $w$ до $\pm \infty$, возьмем интеграл:

$$
\int_{-\infty}^{\infty} \exp \left\{-i\left(E-E\left(\mathbf{q}_{\perp}\right)+g \frac{l+k}{2}\right) w\right\} d w=2 \pi \delta\left(E-E\left(\mathbf{q}_{\perp}\right)+g \frac{l+k}{2}\right) .
$$

Выполняя затем в (20) интегрирование по $E$ и используя выражения (16), в качестве первого приближения асимптотического разложения по $g$ получим

$$
U(k, l)=\frac{a^{2}}{2(2 \pi)^{2}} \operatorname{Re} \int \frac{1+b_{\mathrm{A}}+E\left(\mathbf{q}_{\perp}\right)}{2 E\left(\mathbf{q}_{\perp}\right)} \frac{\cos \left(q_{x}(l-k)\right)}{E\left(\mathbf{q}_{\perp}\right)-b_{(l+k) / 2}+i s} d \mathbf{q}_{\perp},
$$

где $b_{(l+k) / 2}=b+g(l+k) / 2=b_{k}+g(l-k) / 2$ - значение локального внешнего поля в средней между рассматриваемыми спинами точке $(l+k) / 2$. Поскольку малые значения $q_{\perp}^{2}$ дают основной вклад в последний интеграл, для магнонного спектра воспользуемся упрощенным выражением, заменяя исходную зависимость $\gamma_{\mathbf{q}_{\perp}}^{2}$ от $\mathbf{q}_{\perp}$ на получаемую простым разложением по малым значениям $q_{\perp}^{2}$, т. е. запишем

$$
\gamma_{\mathbf{q}_{\perp}}^{2}=\left(\frac{q_{x} / 2+\cos \left(q_{y} / 2\right)+1}{3}\right)^{2} \rightarrow 1-\frac{q_{\perp}^{2}}{12}
$$

и, соответственно, $E\left(q_{\perp}\right) \approx \sqrt{b_{\mathrm{c}}^{2}+q_{\perp}^{2} / 12}$. Далее перейдем к интегрированию по $d \mathbf{q}_{\perp}=q_{\perp} d q_{\perp} d \varphi$, экстраполируем выражение под интегралом на интервал значений $0 \leqslant q_{\perp}<\pi$ и выразим интеграл по углу $\varphi$ через функцию Бесселя первого рода и 


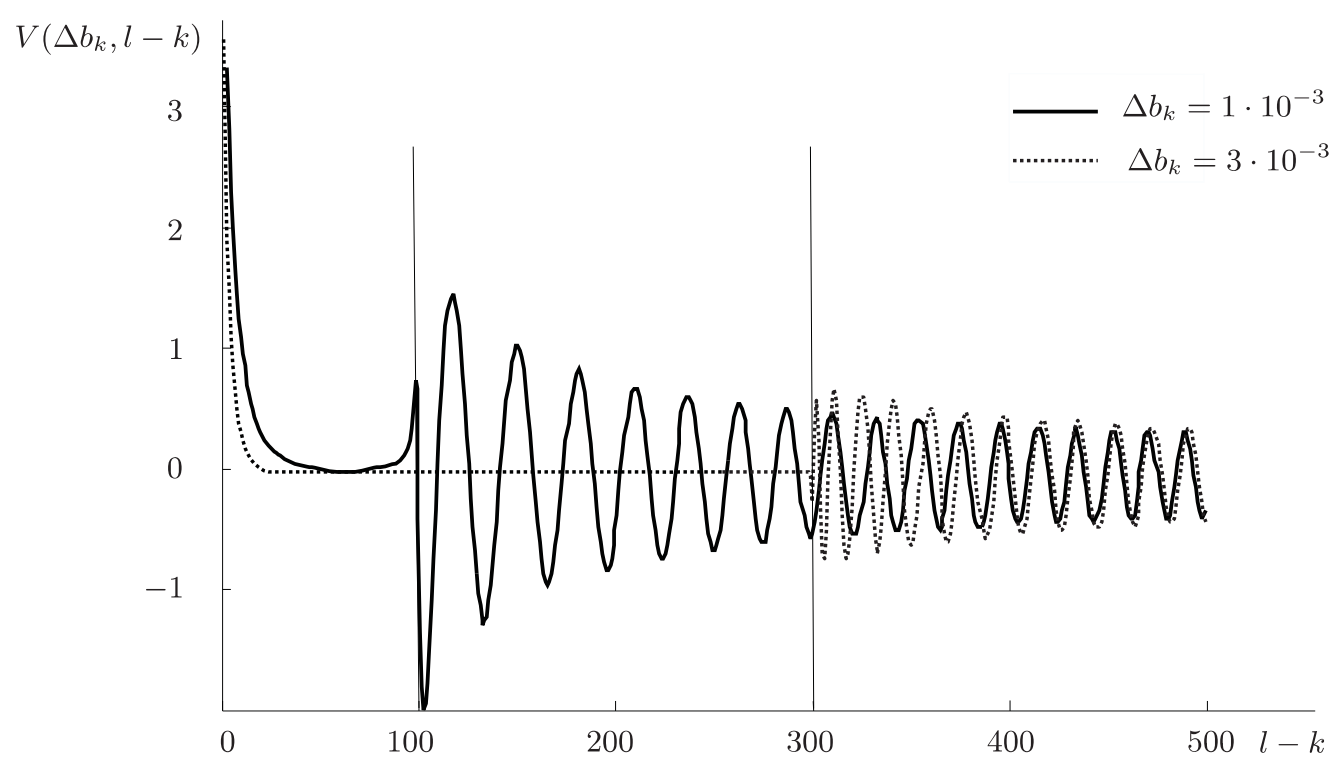

Безразмерное косвенное взаимодействие $V\left(\Delta b_{k}, l-k\right)$ как функция межкубитового расстояния $l-k$, рассчитанное с помощью интегрального выражения (21) для $\Delta b_{k}=1 \cdot 10^{-3}$ и $\Delta b_{k}=3 \cdot 10^{-3}$. Использованы следующие численные значения параметров: $b_{\mathrm{c}}^{2}=1 / 4, g=2 \cdot 10^{-5}, L=100$ и $s=10^{-5}$.

нулевого порядка (см. справочник [10], формула 3.715-18.). В результате получим величину косвенного взаимодействия

$$
\begin{aligned}
\frac{2 \pi}{3 a^{2}} U(k, l)= & V\left(\Delta b_{k}, l-k\right)=\frac{1}{12} \int_{0}^{\pi} \frac{\sqrt{1+b_{\mathrm{c}}^{2}}+\sqrt{b_{\mathrm{c}}^{2}+q_{\perp}^{2} / 12}}{\sqrt{b_{\mathrm{c}}^{2}+q_{\perp}^{2} / 12}} \times \\
& \times \frac{\sqrt{b_{\mathrm{c}}^{2}+q_{\perp}^{2} / 12}-b_{(l+k) / 2}}{\left(\sqrt{b_{\mathrm{c}}^{2}+q_{\perp}^{2} / 12}-b_{(l+k) / 2}\right)^{2}+s^{2}} J_{0}\left(q_{\perp}(l-k)\right) q_{\perp} d q_{\perp} .
\end{aligned}
$$

Пусть локальное внешнее поле $b_{k}=b+g k$ для $k$-го ядерного спина меньше критического значения для ОФП в однородном поле (параметр $\Delta b_{k}=b_{\mathrm{c}}-b_{k}>0$ ), а другой спин $l$ находится на расстоянии $l-k=L n_{l}$ от $k$-го спина, где $L-$ период спинового регистра, $n_{l}=1,2, \ldots$. Заметим, что величина $\sqrt{b_{\mathrm{c}}^{2}+q_{\perp}^{2} / 12}-b_{(l+k) / 2}=$ $\sqrt{b_{\mathrm{c}}^{2}+q_{\perp}^{2} / 12}-b_{\mathrm{c}}+\left(b_{\mathrm{c}}-b_{(l+k) / 2}\right)$ в подынтегральном выражении $(21)$ при $q_{\perp}^{2} \rightarrow 0$ изменяет знак, когда локальное поле в средней точке между рассматриваемыми спинами равно критическому полю, $b_{(l+k) / 2}=b_{\text {c }}$, или при $l-k=L n_{l}=2 \Delta b_{k} / g$. Следствием этого является качественное изменение характера зависимости косвенного взаимодействия от расстояния между спинами, определяемого интегральным выражением (21) (см. рисунок). Как видно из рисунка, зависимость косвенного взаимодействия ядерных спинов от межспинового расстояния качественно различно ведет себя в двух областях: не осциллирует при расстояниях $l-k=L n_{l}<2 \Delta b_{k} / g$ и осциллирует при $l-k=L n_{l}>2 \Delta b_{k} / g$. 
Сохраняя в знаменателе (21) только члены, квадратичные по $\mathbf{q}_{\perp}$, получим приближенное выражение (см. справочник [10], формулы 6.532-4, 9.561-5):

$$
\begin{aligned}
\frac{2 \pi}{3 a^{2}} U(k, l) & =V\left(\Delta b_{k}, l-k\right) \approx 2\left(\sqrt{1+b_{\mathrm{c}}^{2}}+b_{\mathrm{c}}\right) \int_{0}^{\infty} \frac{J_{0}\left(\mathbf{q}_{\perp}(l-k)\right)}{\mathbf{q}_{\perp}^{2}+\mu_{(l+k) / 2}^{2}} \mathbf{q}_{\perp} d \mathbf{q}_{\perp}= \\
& =2\left(\sqrt{1+b_{\mathrm{c}}^{2}}+b_{\mathrm{c}}\right) K_{0}\left(\mu_{(l+k) / 2}|l-k|\right),
\end{aligned}
$$

где $K_{0}(x)$ - функция Макдональда нулевого порядка и $\mu_{(l+k) / 2}^{2}=24 b_{\mathrm{c}}\left(b_{\mathrm{c}}-b_{(l+k) / 2}\right)>0$. Как видно из выражения $(22)$, величина $\left(\mu_{(l+k) / 2}\right)^{-1}$ представляет собой эффективный радиус косвенного взаимодействия между ядерными спинами. В случае однородного поля он быстро увеличивается вблизи критического значения поля, соответствующего точке ОФП, когда $b \rightarrow b_{c}$. В случае неоднородного поля это происходит, только когда локальное поле в средней точке для рассматриваемых двух спинов становится близким к критическому значению: $b_{(l+k) / 2} \rightarrow b_{c}$. Положение средней точки между двумя ядерными спинами $b_{c}=b_{(l+k) / 2}$, в которой параметр $\mu_{(l+k) / 2}^{2}$ изменяет знак, будем называть “точкой поворота" по аналогии с задачей отражения от барьера квантовой частицы в квазиклассическом приближении [11]. Чтобы получить вещественное значение $V\left(\Delta b_{k}, l-k\right)$ для случая $\mu_{(l+k) / 2}^{2}=-v_{(l+k) / 2}^{2}<0$, примем в качестве нижнего предела в интеграле в $(22)$ величину $v_{(l+k) / 2}>0$. В результате мы получим (см. справочник [10], формулы 8.405-1, 8.407-2)

$$
V\left(\Delta b_{k}, l-k\right) \approx-2\left(\sqrt{1+b_{\mathrm{c}}^{2}}+b_{\mathrm{c}}\right) \frac{\pi}{2} N_{0}\left(\nu_{(l+k) / 2}(l-k)\right)
$$

где $N_{0}(x)$ - осциллирующая функция Неймана (или функция Вебера $\left.Y_{0}(x)\right)$ нулевого порядка. Эти приближенные выражения хорошо согласуются с результатами численного интегрирования, представленными на рисунке.

\section{6. ЗАКЛЮЧЕНИЕ}

В настоящее время в мире проявляется исключительный интерес к разработкам различных вариантов квантовых компьютеров вообще и, в частности, к разработкам ЯМР-квантовых компьютеров, в которых в качестве квантовых битов (кубитов) предполагается использовать отдельные ядерные спины атомов в твердотельных структурах. Существенно, что для организации квантовых вычислений важное значение имеет возможность управления взаимодействием не только между соседними, но и между удаленными ядерными спинами в регистре. Примером такого взаимодействия является рассматриваемое в предлагаемой статье косвенное взаимодействие между ядерными спинами за счет сверхтонкого взаимодействия ядерных спинов со спиновыми волнами в одноосной антиферромагнитной пластине.

Отметим, что косвенное взаимодействие между ядерными спинами в одноосном антиферромагнетке с неоднородным внешним полем в условиях однородного антиферромагнитного резонанса, механизмы продольной и поперечной релаксации состояний отдельных ядерных спинов, а также процессы декогерентизации парных запутанных состояний ядерных спинов были изучены нами в работах [6], [12]. 


\section{ПРИЛОЖКЕНИЕ}

После преобразования коммутатора в выражении $(18)$ найдем $\mathbf{h}_{\mathrm{II}}(k, l)$ :

$$
\begin{aligned}
& \mathbf{h}_{\mathrm{II}}(k, l) \approx-i \frac{a^{2}}{4} \int_{-\infty}^{0}\left\{I^{+}(k) I^{-}(l)\left\langle 0\left|\left[S^{-}(\tau, k), S^{+}\left(\tau+\tau^{\prime}, l\right)\right]\right| 0\right\rangle+\right. \\
& \left.\quad+I^{+}(l) I^{-}(k)\left\langle 0\left|\left[S^{-}(\tau, l), S^{+} t\left(\tau+\tau^{\prime}, k\right)\right]\right| 0\right\rangle\right\} e^{s \tau^{\prime}} d \tau^{\prime}+\text { э. c. }- \\
& \quad-i \frac{a^{2}}{4} \sum_{j=k, l} \int_{-\infty}^{0}\left\{\frac{1}{2}\left\langle 0\left|\left[S^{-}(\tau, j), S^{+}\left(\tau+\tau^{\prime}, j\right)\right]\right| 0\right\rangle+\right. \\
& \left.\quad+I_{z}(j)\left\langle 0\left|S^{-}(\tau, j) S^{+}\left(\tau+\tau^{\prime}, j\right)+S^{+}\left(\tau+\tau^{\prime}, j\right) S^{-}(\tau, j)\right| 0\right\rangle\right\} e^{s \tau^{\prime}} d \tau^{\prime}+\text { э. c. },
\end{aligned}
$$

где было учтено, что

$$
\begin{aligned}
I^{ \pm}(j) I^{\mp}(j) & =\frac{1}{2} \pm I_{z}(j), & {\left[I^{ \pm}(j), I^{\mp}(j)\right] } & = \pm 2 I_{z}(j), \\
I_{z}(j) I^{ \pm}(j) & =-I^{ \pm}(j) I_{z}(j)= \pm \frac{1}{2} I^{ \pm}(j), & & {\left[I_{z}(j), I^{ \pm}(j)\right]= \pm I^{ \pm}(j) . }
\end{aligned}
$$

Среднее значение по основному состоянию коммутатора магнонных операторов (7) при $\tau=0$ равно

$$
\left\langle 0\left|\left[\xi\left(q_{y}, E\right), \xi^{+}\left(q_{y}^{\prime}, E^{\prime}\right)\right]\right| 0\right\rangle=\left\langle 0\left|\xi\left(q_{y}, E\right) \xi^{+}\left(q_{y}^{\prime}, E^{\prime}\right)\right| 0\right\rangle=\delta\left(q_{y}-q_{y}^{\prime}\right) \delta\left(E-E^{\prime}\right),
$$

отсюда получим среднее значение коммутатора

$$
\begin{gathered}
\langle 0|\left[S^{-}(\tau, k), S^{+}(\tau\right. \\
\left.\left.+\tau^{\prime}, l\right)\right]|0\rangle=\frac{1}{(2 \pi)^{2}} \int\left\{-u^{*}\left(\mathbf{q}_{\perp}, E\right) u\left(q_{x}^{\prime}, q_{y}, E\right) e^{-i E_{-} \tau^{\prime}+s \tau^{\prime}}+\right. \\
\left.+v\left(\mathbf{q}_{\perp}, E\right) v^{*}\left(q_{x}^{\prime}, q_{y}, E\right) e^{i E_{+} \tau^{\prime}+s \tau^{\prime}}\right\} e^{i\left(q_{x} k-q_{x}^{\prime} l\right)} d E d \mathbf{q}_{\perp} d q_{x}^{\prime}
\end{gathered}
$$

и среднее значение суммы произведений спиновых операторов

$$
\begin{aligned}
&\langle 0| S^{-}(\tau, j) S^{+}\left(\tau+\tau^{\prime}, j\right)+S^{+}\left(\tau+\tau^{\prime}, j\right) S^{-}(\tau, j)|0\rangle= \\
&= \frac{1}{(2 \pi)^{2}} \int\left\{u^{*}\left(\mathbf{q}_{\perp}, E\right) u\left(q_{x}^{\prime}, q_{y}, E\right) e^{-i E_{-} \tau^{\prime}+s \tau^{\prime}}+\right. \\
&\left.\quad+v\left(\mathbf{q}_{\perp}, E\right) v^{*}\left(q_{x}^{\prime}, q_{y}, E\right) e^{i E_{+} \tau^{\prime}+s \tau^{\prime}}\right\} e^{i\left(q_{x}-q_{x}^{\prime}\right) j} d E d \mathbf{q}_{\perp} d q_{x}^{\prime} .
\end{aligned}
$$

Используя эти результаты в выражении (П.1), после громоздких, но простых преобразований и интегрирования по $\tau^{\prime}$, получим полный гамильтониан двух ядерных спинов атомов $k$ и $l$, принадлежащих одной подрешетке $\mathbf{A}$ :

$$
\begin{aligned}
\mathbf{h}_{\mathrm{II}}(k, l) & =-\sum_{j=k, l}\left(\omega_{\mathrm{I}}(j)-\frac{a}{2}-W(j)\right) I_{z}+\Delta \mathbf{h}_{\mathrm{II}}(k, l), \\
\Delta \mathbf{h}_{\mathrm{II}}(k, l) & =-U(k, l)\left(I^{-}(k) I^{+}(l)+I^{+}(k) I^{-}(l)\right),
\end{aligned}
$$

где

$$
\begin{aligned}
U(k, l)= & \frac{a^{2}}{2(2 \pi)^{2}} \operatorname{Re} \int\left\{\frac{u^{*}\left(q_{x}, q_{y}, E\right) u\left(q_{x}^{\prime}, q_{y}, E\right)}{E_{-}+i s}-\frac{v\left(q_{x}, q_{y}, E\right) v^{*}\left(q_{x}^{\prime}, q_{y}, E\right)}{E_{+}-i s}\right\} \times \\
& \times e^{i\left(q_{x} k-q_{x}^{\prime} l\right)} d E d \mathbf{q}_{\perp} d q_{x}^{\prime}
\end{aligned}
$$


- выражение для косвенного взаимодействия между поперечными составляющими ядерных спинов, принадлежащими одной и той же подрешетке, обобщающее известное выражение Накамуры для однородного поля [1], а

$$
\begin{aligned}
W(j)= & \frac{a^{2}}{2(2 \pi)^{2}} \operatorname{Re} \int\left\{\frac{u^{*}\left(q_{x}, q_{y}, E\right) u\left(q_{x}^{\prime}, q_{y}, E\right)}{E_{-}+i s}+\frac{v\left(q_{x}, q_{y}, E\right) v^{*}\left(q_{x}^{\prime}, q_{y}, E\right)}{E_{+}-i s}\right\} \times \\
& \times e^{i\left(q_{x}-q_{x}^{\prime}\right) j} d E d \mathbf{q}_{\perp} d q_{x}^{\prime}
\end{aligned}
$$

- поправка к локальной частоте ядерного магнитного резонанса.

Благодарности. Авторы благодарны академику К. А. Валиеву за поддержку и внимание к работе. Работа была поддержана РФФИ (проекты № 05-02-17412_а, 06-07-89129_а и 08-07-00481_а).

\section{Список литературы}

[1] T. Nakamura, Progr. Theor. Phys., 20:4 (1958), 542-552.

[2] Е.А. Туров, М.П. Петров, Ядерный магнитный резонанс в ферро- и антиферромагнетиках, Наука, М., 1969.

[3] М. И. Куркин, Е. А. Туров, ЯМР в магнитоупорядоченных веществах и его применения, Наука, М., 1990.

[4] А.А. Кокин, Твердотельные квантовые компьютеры на ядерных спинах, ИКИ, М., Ижевск, 2004.

[5] A. A. Kokin, Proc. SPIE, 6264 (2006), 626407.

[6] A. A. Kokin, V. A. Kokin, Proc. SPIE, 7023 (2008), 70230B, arXiv: 0812.0135.

[7] С. В. Тябликов, Методы квантовой теории магнетизма, Наука, М., 1975.

[8] А.Х. Найфе, Методъ возмущений, Мир, М., 1976.

[9] А. Г. Гуревич, Магнитный резонанс в ферритах и антиферромагнетиках, Наука, М., 1973.

[10] И. С. Градштейн, И.М. Рыжик, Таблицы интегралов, сумм, рядов и произведений, Физматлит, М., 1963.

[11] Л.В. Ландау, Е.М. Лифшиц, Квантовая механика (нерелятивистская теория), Наука, М., 1974.

[12] A. A. Kokin, V. A. Kokin, Quantum Computers and Computing, 9:1 (2009), 19-37, arXiv: 1003.0170.

Поступила в редакцию 27.02.2011, после доработки 27.03.2011 